\title{
Étude de la mise en régime des écoulements sur les ouvrages à forte pente
}

\author{
Application au problème de l'entrainement d'air
}

\author{
Study of the setting up of the flow regime \\ on high gradient structures \\ Application to air entrainment problem
}

PAR G. HALBRONN

INGENHEUR DES PONTS ET CHAUSSÉES

\begin{abstract}
Etude thérique de l'ecoulement à surface libre d'un fluide sur un coursier long, compte tenu de l'effet de la viscosité; calcul des vitesses locales et des profondeurs en régime laminaire et turbulent; existence d'un point critique où la turbulence commence à faire sentir son action en surface et à partir duquel l'entrainement d'air devient possible. - Vérifications expérimentales. - Etude schématique du critère d'apparition de l'eau blanche en aval du point critique.
\end{abstract}

\begin{abstract}
Theoretical study of free surface. fluid flow over a long spillway, account being taken of viscosity.

Calculation of local velocities and depths in laminary and turbulent flow regimes. Existance of a critical point where turbulent action becomes noticeable on the surface and air entrainment becomes possible. Experimental verification. Schematic study of criterion of appearance of "white water" below critical point.
\end{abstract}

\section{AVANT-PROPOS}

Le travail présenté ici fait partie d'une étude plus vaste sur l'entraînement d'air effectuée avec une importante contribution financière de la Direction des Etudes et Recherches de l'Electricité de France, étude qui nous a été confiée par M. Nizerì, Directeur du Service des Etudes et Recherches hydrauliques. Celui-ci a bien voulu nous communiquer également les résultats nécessaires pour appliquer notre théorie aux belles expériences réalisées au Laboratoire National d'Hydraulique sur le modèle du déversoir de Bort-lesOrgues.

Le Laboratoire Dauphinois d'Hydraulique nous a offert l'hospitalité de sa Station d'Essai et les ressources de sa vaste documentation, tout en assumant le surplus de la charge financière. II nous est agréable de devoir en remercier MM. GA-
RIEL et Dagallier, Directeurs des Etablissements Neyrpic, et spécialement M. DANEL, Directeur du Laboratoire, Professeur à l'Institut Polytechnique de Grenoble, qui nous a signalé l'intérêt de cette recherche et nous a fourni la plupart des idées de base de l'étude théorique.

La Faculté des Sciences de Grenoble a bien voulu s'intéresser à notre travail et l'accepter comme première thèse pour l'attribution du titre d'Ingénieur-Docteur. Nous tenons particulièrement $\dot{a}$ exprimer notre reconnaissance à M. Esclangon, Professeur à la Faculté des Sciences, Directeur de l'Ecole Nationale Supérieure d'Ingénieurs, ainsi qu’à MM. Kravtchenko, Professeur à la Faculté des Sciences, et Santon, Maître de Conférences, qui ne nous ont ménagé ni l'appui de leurs conseils, ni le secours de leur érudition.

\section{INTRODUCTION}

\section{NATURE DU PHÉNOMENE ETUDIE}

Les écoulements sur les seuils déversants ont fait l'objet de nombreuses études, tant théoriques qu'expérimentales, dans le but de déterminer, en fonction du tracé, le coefficient de débit, la ré- partition des pressions sur le fond et même la distribution des vitesses. Ces études ont montré que les phénomènes sont bien re] par les lois des écoulements à potenti 
tesse des fluides parfaits. Lorsque le tracé de la surface libre est connu, des méthodes graphiques classiques permettent d'obtenir les valeurs des vitesses et des pressions en chaque point; si la ligne d'eau est inconnue, il est encore possible par tâtonnements de se ramener au premier cas.

La validité de ces conclusions est cependant limitée à des ouvrages courts. Considérons, pour préciser ce point, un seuil déversant galbé très large, alimenté de telle sorte que l'eau l'aborde sans perturbation et prolongé vers l'aval par un coursier plan d'inclination constante; sur un tel déversoir, la vitesse d'un fluide parfait croìtrait indéfiniment, alors qu'en réalité un régime uniforme tend à s'établir.

L'explication de ce désaccord se trouve évidemment dans les effets de la viscosité; pour un fluide réel, la vitesse est nulle au contact de la paroi solide; elle atteint la valeur qui lui est assignée par l'écoulement à potentiel à une certaine distance du fond qui détermine l'épaisseur de la couche dite " couche limite» où se concentre pratiquement la dissipation d'énergie due à la viscosité. De hauteur très faible près de la crête, ce qui justifie le mode d'étude au voisinage de cette ligne par la mécanique des fluides parfaits, cette couche (qui peut ètre laminaire) augmente d'importance vers l'aval, devient en général turbulente et parvient à occuper toute la hauteur de l'écoulement. Au-delà, la distribution des vitesses peut encore se modifier pour tendre vers celle du régime uniforme où l'effet accélérateur de la pesanteur compense exactement l'effet retardateur de la viscosité.

L'observation courante d'un modèle de laboratoire s'accorde avec cette description. En passant sur la crête déversante, les couches superficielles du liquide présentent une surface bien lisse (mises à part de petites rides temporaires); la surface du déversoir apparaît comme si l'eau était immobile, lorsqu'elle est suffisamment claire. Cette transparence se prolonge à quelque distance en aval, distance qui dépend de la profondeur (donc du débit et de la pente), de l'état de surface du déversoir et de la tranquillité de l'alimentation. Brusquement, la surface libre cesse d'être transparente; elle semble rugueuse, couverte de petites rides changeantes. Le point où apparaît ce phénomène n'est pas, il est vrai, rigoureusement fixe, mais il oscille autour d'une position moyenne que, pour abréger, nous appelons « point critique».

Ces fluctuations font pressentir le lien entre l'existence du point critique et les phénomènes de turbulence. Un des objets de notre étude sera de montrer que ce point où la surface libre change d'aspect est précisément celui où la turbulence créée à l'interface eau-paroi fait sentir son influence jusqu'à la surface; c'est dire qu'alors la couche limite turbulente atteint une hauteur égale à la profondeur de l'écoulement.

Dans la nature, il se produit également au voisinage d'un point fixe un phénomène nouveau qui influe sur le fonctionnement des évacuateurs; il s'agit de «l'eau blanche » due à l'entrainement d'air dans la masse. Les écoulements habituels à faible pente présentent une surface de séparation nette entre l'air, milieu ambiant, et le liquide; au contraire, lorsque l'eau blanche existe, il n'y a plus de limite bien tranchée entre les domaines des deux phases. Tandis que des gouttelettes sont projetées à de grandes hauteurs, des bulles gazeuses pénètrent dans le sein de la masse liquide et s'écoulent avec elle, lui conférant un aspect laiteux caractéristique. Nous nous proposons de montrer que l'eau blanche ne peut apparaitre en amont du point critique.

En effet, nous avons dit qu'en ce point les fluctuations turbulentes commencent à faire sentir leur effet en surface. Entre autres, elles impriment aux particules superficielles des vitesses transversales qui, dans certains cas, sont suffisantes pour leur permettre de s'échapper de la masse sous forme de gouttes qui retombent plus loin en entraînant avec elles de l'air, par compression à l'avant et par frottement sur les côtés.

Ce mode d'explication rend compte du fait que, au contact des parois latérales, l'eau blanche se produit dès la crête du déversoir et sur une largeur croissante vers l'aval, visualisant ainsi la couche où existe la turbulence. Si le coursier est étroit, les deux zones latérales d'entraînement d'air finissent par se rejoindre dans l'axe et ce sont elles qui donnent alors naissance au phénomène généralisé à toute la masse que nous décrivions plus haut. De même, un obstacle isolé sur le fond accroît la turbulence à son voisinage et peut donner lieu à un entrainement d'air local.

Si l'eau blanche est susceptible de se produire a partir du point critique, ellc n'y apparait pas pour autant dans tous les cas. Il est encore nécessaire que l'énergie cinétique transversale conférée par la turbulence aux particules superficielles soit suffisante peur vaincre les effets stabilisateurs dus en particulier aux forces de capillarité. On explique ainsi que l'entraînement d'air constaté sur un ouvrage n'apparaisse pas toujours sur un modèle en similitude de Froude réduit y. fois, car l'énergie cinétique transversale des gouttes d'eau est alors divisée par $\mu^{4}$, alors que le travail des forces de tension superficielle lors de l'éjection de cette goutte est divisé seulement par y.2. La condition exprimant la possibilité de l'arrachement d'une particule superficielle pourra donc être remplie dans la réalité sans l'être sur le modèle. 
Si cette condition n'est pas vérifiée, les particules seront incapables de s'échapper et se contenteront de bosseler plus ou moins la surface, lui conférant la forte rugosité que nous avons déjà mentionnée. Dans ce cas, l'air sera entrainé au voisinage de la surface libre et à partir du point critique, par un mécanisme analogue a celui d'une pompe à viscosité. II est nécessaire de bien distinguer cet entraînement d'air sans mélange des fluides de l'eau blanche qui se produit lorsque le critère, dont nous laissons prévoir l'existence ci-dessus, est rempli.

L'étude qui va suivre sera divisée en trois parties. Dans la première, nous établirons théoriquement les équations générales régissant l'évolution de la couche limite sur un coursier infiniment large, équations qui permettent l'étude détaillée de l'écoulement en amont du point eritique et même en aval lorsque l'eau blanche ne s'y produit pas. Nous discuterons plus spécialement ces équations dans le cas important oì la couche limite est turbulente et où le coursier devient plan en aval du déversoir galbé.

La deuxième partie sera consacrée à la comparaison de ces résultats théoriques et des résultats expérimentaux dont nous disposons. Une série d'essais effectués par nos soins au Laboratoire Dauphinois d'Hydraulique sur un modele réduit met en évidence un bon accord dans le cas le plus simple d'un déversoir lisse, très large, constitué d'un seuil galbé prolongé par un coursier plan. Une seconde série d'expériences, réalisée au Laboratoire National d'Hydrauficue, correspond à un dispositif plus complexe : parois rugueuses, coursier gauche, bajoyers rapprochés et convergents; la confrontation porte uniquement sur la position du point critique. Enfin, des expériences effectuées aux Etats-Unis et limitées elles aussi à la détermination des conditions au point critique seront discutées ensuite. Elles ont le grand intérèt de comporter des observations dans la nature où apparait l'eau blanche.

Dans la troisième partie, nous aborderons l'étude des déformations de la surface libre en aval du point critique. Malheureusement, la mise en équation de ce probleme est complexe; elle suppose la connaissance de la structure de la turbulence superficielle, de l'action du vent relatif, des effets de la capillarité et des pressions...

Nous nous contenterons de présenter une tentative très grossière dans la voie de la recherche du critérium d'instabilité de la surface libre sous l'effet de ces différents facteurs. Elle aura peut-être néanmoins l'intérêt de préciser les cau- ses qui engendrent le phénomene, le sens dans lequel elles agissent, les groupements sans dimension qui s'introduisent dans l'étude, sans qu'il faille rechercher la rigueur dans le mode de déduction ni l'accord numérique entre les rísultats et un contrôle expérimental que nous n'avons pas encore été à même de réaliser complètement.

En résumé, nous étudions l'écoulement sur un déversoir très large. Si l'ouvrage est court, la couche limite turbulente n'atteint pas la surface, il ne se produit pas d'entraînement d'air et l'écoulement peut être étudié complètement au moyen des formules données plus loin. Par contre, si le point critique est situé sur l'ouvrage, l'eau blanche est susceptible d'apparaitre à partir de ce point si un certain critere, dont nous cherchons à donner une idée, est vérifié. Sinon, la surface devient simplement rugueuse et l'air est entrainé par frottement sans mélange des phases.

L'entrainement d'air, jusqu'à ces dernières années, est demeuré un sujet peu étudié, quoique de nombreux auteurs $[1],[2],[3],[4]^{1}$ aient signalé les dangers que pouvait faire courir la méconnaissance de ses lois dans l'implantation des canaux d'évacuation à forte pente, des bassins de tranquillisation, etc... De plus, les conditions dans lesquelles il se produit sembaient déroutantes; Schorlitsch [5] avait cru pouvoir conclure à l'existence d'une vitesse critique d'apparition; cette opinion a été combattue par la suite sur la foi d'observations contraires [1]. La différence de comportement des ouvrages et des modèles a également frappé les expérimentateurs [6]. Nous espérons que notre exposé fera mieux comprendre la genc̀se du phénomène; si des travaux ultérieurs sont nécessaires pour connaître les lois de l'écoulement mixte, la méthode de calcul proposée ici s'appliquera à l'étude du mouvement en amont du point critique, et en aval lorsque l'eau blanche n'apparait pas. Par une détermination précise des profondeurs et des vitesses en chaque point, elle permettra une prévision plus exacte des hauteurs nécessaires des bajoyers, du tracé optimum des courbes dans le plan vertical, une appréciation des vitesses terminales qui conditionnent l'établissement des ouvrages de dissipation d'énergie. L'évaluation des forces de frottement sur le fond permettra de connaitre l'efrort de renversement exercé par l'eau en mouvement sur le déversoir, qui peut ne pas être négligeable aux gros débits.

1. Les chiffres entre crochets renvoient à la bibliographis. 
INDEX DES NOTATIONS

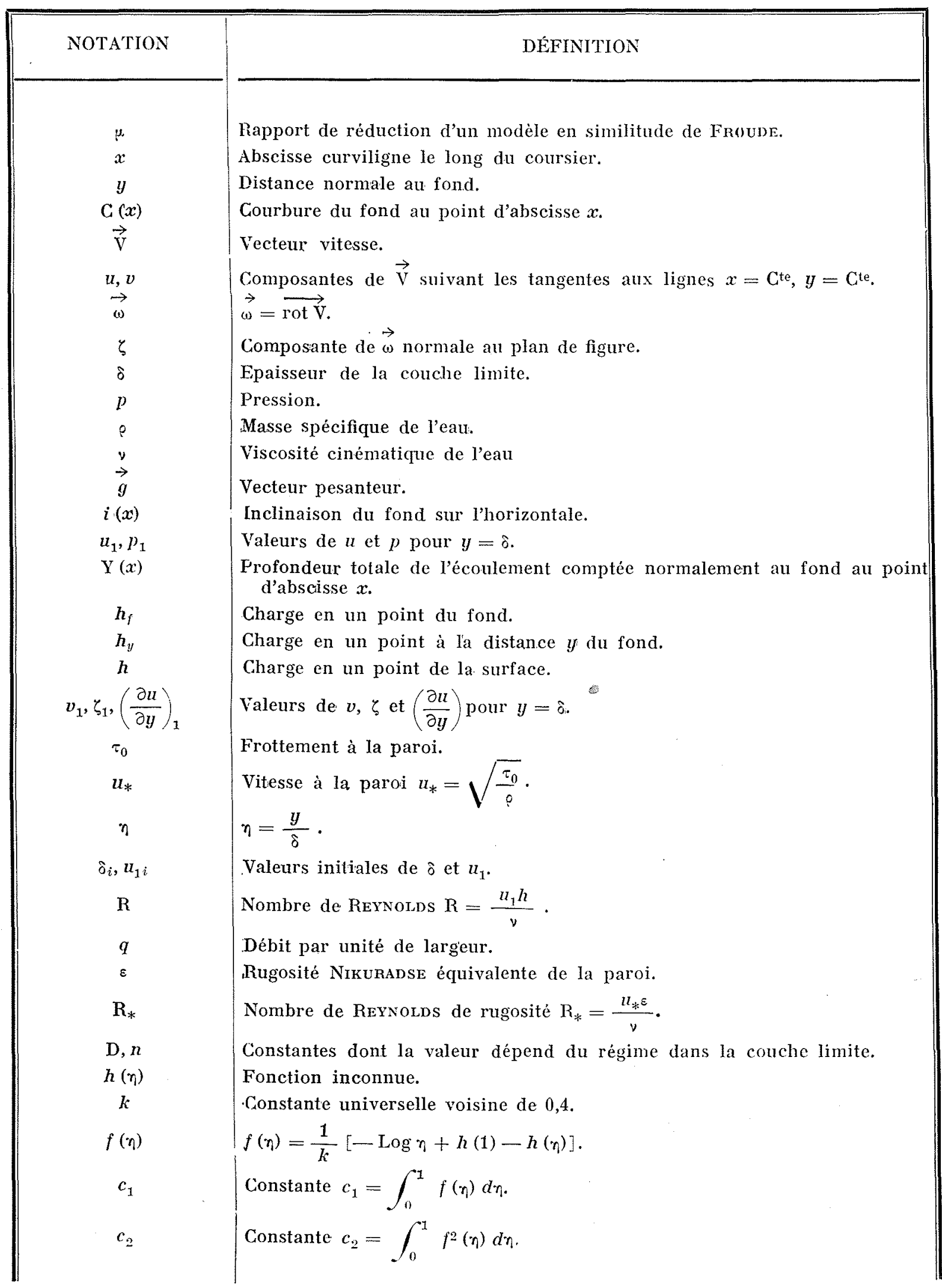




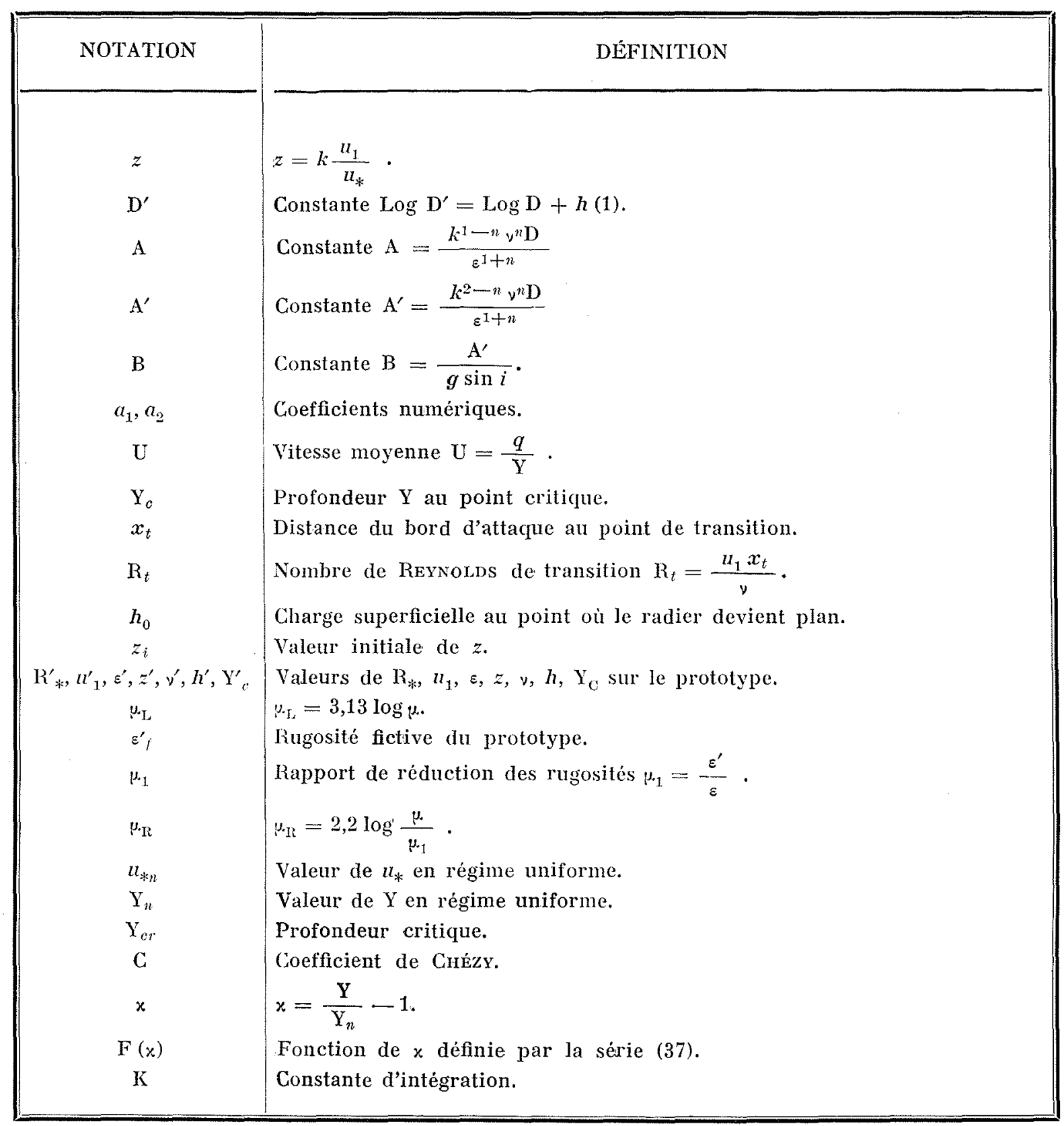


PREMIERE PARTIE

\title{
ÉTUDE THÉORIQUE DE L'ÉCOULEMENT D'UN FLUIDE VISQUEUX SUR UN COURSIER COUCHE LIMITE BIDIMENSIONNELLE SOUMISE A LA PESANTEUR
}

\author{
CHAPITRE I \\ ÉQUATIONS GÉNÉRALES DU MOUVEMENT
}

\section{I. - Établissement des équations}

de la couche limite le long d'une paroi courbe, compte tenu de la pesanteur.

Nous traiterons le cas d'un coursier suffisamment large pour que, dans l'axe, le phénomène soit peu modifié par la présence des parois latérales. La coordonnée normale au plan de l'écoulement n'intervient done pas, et le probleme est à deux dimensions.

L'établissement des équations de la couche limite le long d'une paroi courbe, à partir des équations de Navier écrites en coordonnées curvilignes orthogonales, et un problème classique [7].

Nous les rétablirons rapidement dans le cas particulier actuel, suivant la méthode de PrandTL.

Soient des coordomnes orthogonales directes dont les lignes $x=\mathrm{C}^{\text {te }}$ sont les normales à la paroi et les lignes $y=C^{\text {to }}$ les courbes parallèles à la paroi. Si d'un point $\mathrm{M}(x, y)$ nous abaissons la normale $\mathrm{Mm}$ sur le fond, $y$ est mesuré par la longueur $\overline{m M}$ et $x$ par l'abseisse curviligne de $m$, mesurée le long de la paroi à partir d'un point fixe $\mathrm{O}$. C, fonction de $x$, désignant la courbure du

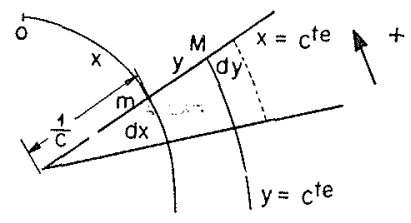

Fig. 1.

fond en $m$, comptée positivement lorsque le centre de courbure est situé du côté des $y$ négatifs, les éléments d'are le Iong des lignes de coordonnées sont évidemment $(1+-\mathrm{C} l y) d x$ et $d \eta$. Représentons par $\vec{V}$ le vecteur vitesse de composantes $u, v$; seule la composante $\zeta$ normale au plan de figure du vecteur $\vec{\omega}=\overrightarrow{\operatorname{rot} V}$ n'est pas nulle. La formule de Rremann permet d'écrire :

$$
\zeta(1+C y)=-\frac{\partial}{\partial y}[u(1+C y)]+\frac{\partial v}{\partial x}
$$

oll :

$$
\zeta=-\frac{\partial u}{\partial y}-\frac{\mathrm{C}}{1+\mathrm{C} y} u+\frac{1}{1+\mathrm{C} y} \frac{\partial v}{\partial x}
$$

De même, d'après la formule de SToriss, l'équation de continuité div $\vec{v}=0$ s'écrit :

$$
\frac{\partial u}{\partial x}+\frac{\partial}{\partial y}[v(1+\mathrm{C} y)]=0
$$

ou :

$$
\frac{\partial u}{\partial x}+(1+\mathrm{C} y) \frac{\partial y}{\partial y}+\mathrm{C} v=0 .
$$

Si o désigne alors l'épaisseur de la couche limite, $u$ varie de la valeur 0 à la paroi à sa valeur $u_{1}$ au bord de la couche limite sur la longueur $\delta$; $u_{1}$ étant pris comme étalon de grandeur pour les vitesses et ó étant supposé petit, $\frac{\partial u}{\partial y}$ est d'ordre $\delta^{-1}$ et $\frac{\partial^{2} u}{\partial y^{2}}$ d'ordre $\delta^{-2}$ dans la couche limite. De même $u, \frac{\partial u}{\partial x}, \frac{\partial^{2} u}{\partial x^{2}}$ sont d'ordre $\delta^{0}$.

L'équation de continuité peut s'écrire :

$$
v(1+\mathrm{C} y)=\int_{0}^{y} \frac{\partial u}{\partial x} d y \quad 0 \leqslant y \leqslant \delta
$$

ce qui montre que, sauf peut-être en un point de rebroussement où $C$ serait infini, $v$ est infiniment petit d'ordre o. Dans ce cas, $\frac{\partial v}{\partial x}$ et $\frac{\partial^{2} v}{\partial x^{2}}$ sont d'ordre $\delta, \frac{\partial v}{\partial y}$ est d'ordre $\delta^{0}, \frac{\partial^{2} v}{\partial y^{2}}$ d'ordre $\delta^{-1}$.

D'autre part, l'équation vectorielle du mouvement permanent s'écrit :

$-\overrightarrow{\mathrm{V}} \wedge \vec{\omega}=-\overrightarrow{\operatorname{grad}}\left(\frac{p}{p}+\frac{1}{2}|\overrightarrow{\mathrm{V}}|^{2}\right)+\vec{g}-\nu \overrightarrow{\operatorname{rot} \omega}(2)$

$p$ désignant la pression en un point, $q$ et, la masse spécifique et la viscosité cinématique de l'eau et $\vec{g}$ le vecteur pesanteur d'intensité $g$ et de direction verticale descendante. 


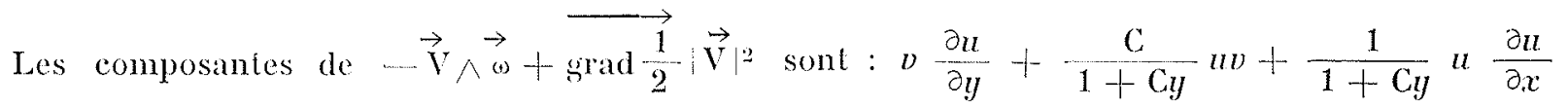

$$
\text { et }: \quad-\frac{\mathrm{C}}{1+\mathrm{C} y} u^{2}+\frac{1}{1+\mathrm{C} y} u \frac{\partial v}{\partial x}+v \frac{\partial v}{\partial y}
$$

dont les termes principaux sont $u \frac{\partial u}{\partial x}+v \frac{\partial u}{\partial y}$ et - Cü, puisque Cy est supposé de l'ordre de $\tilde{\partial}$.

Les termes négligés sont au moins dordre Cò pour la première composante et ó pour la seconde.

D'après la formule de Riemans, on obtient comme ci-dessus les composantes de $\overrightarrow{\text { rot } \omega}$ :

et :

$$
\frac{\partial \zeta}{\partial y}=-\frac{\partial^{2} u}{\partial y^{2}}-\frac{\mathrm{C}}{1+\mathrm{C} t y} \frac{\partial u}{\partial y}+\frac{\mathrm{C}^{2}}{(1+\mathrm{C} y)^{2}} u+\frac{1}{1+\mathrm{C} y} \frac{\partial^{2} v}{\partial x \partial y}-\frac{\mathrm{C}}{(1+\mathrm{C} y)^{2}} \frac{\partial v}{\partial x}
$$

$\left.-\frac{1}{1+\mathrm{C} y} \frac{\partial \zeta}{\partial x}=-\frac{1}{1+\mathrm{C} y} \mid-\frac{\partial^{2} u}{\partial x^{2} \partial y}-\frac{\mathrm{C}}{1+\mathrm{C} y} \frac{\partial u}{\partial x}-\frac{1}{(1+\mathrm{C} y)^{2}} \frac{d \mathrm{C}}{d x} u-\frac{1}{1+\mathrm{C} y} \frac{1}{\partial x^{2}}-\frac{\partial^{2} v}{(1+\mathrm{C} y)^{2}} \frac{d^{2}}{\partial x} \frac{\partial v^{-}}{\partial x}\right]$

dont les termes principaux sont -- $\frac{\partial^{2} u}{\partial y^{2}}$ et $\frac{\partial{ }^{2} u}{\partial x \partial y}$, a la condition que $\partial^{\prime 2} \frac{d \mathrm{C}}{d x}$ soit fini ou infiniment petit. Les équations risqueraient done de tomber en défaut en un point où $C$ varie brusquement. Les termes négligés sont au moins d'ordre Cón pour la première composante, $\mathrm{C}$ et $\frac{d \mathrm{C}}{d x}$ pour la seconde.

Finalement, la projection suivant $O x$ de l'équation vectorielle (2) s'écrit :

$u \frac{\partial u}{\partial x}+v \frac{\partial u}{\partial y}=-\frac{1}{i} \frac{\partial p}{\partial x}+g \sin i+v \frac{\partial-u}{\partial y^{2}}$

$i$, fonction de $x$, désignant l'inclinaison du fond sur l'horizontale comptée positivement vers le bas.

Par hypothese, dans la couche limite, les termes de viscosité sont de l'ordre de grandeur des

1. Lintroduction du leme - Che est due al docteur Gorbstens. Il faut cependant jemarquer que l'équation correspondante contient des termes en $\delta^{-1}$ el $\delta^{0}$; rien n'est done clangé, ainsi que nous venons de te voil, si on la conserve sous la forme plus simple:

$$
-{ }^{1} \frac{\partial p}{\partial} \frac{\partial y}{} \quad=0
$$

l.a métlode de Vor Mrses [8] conduit d'ailleurs à ce même résultat. Cet auteur considère des variables sans dimension, exprimées en fonction d'une longueur carac- termes d'inertie; l'équation (3) montre alors que o est de l'ordre de $v^{1 / 2} ;$; elle est donc vérifiée, à des termes d'ordre Cồ près. La projection de (2) sur Oy s'écrit de mème :

$$
\frac{1}{i} \frac{\partial p}{\partial y}=g \cos i-\mathrm{C} u^{2}
$$

à des termes d'ordre ô près.

Il faut y adjoindre l'équation de continuité :

$$
\frac{\partial u}{\partial x}+\frac{\partial v}{\partial y}=0
$$

à des termes d'ordre Cồ près.

Les équations du mouvement sont done idenliques à celles de la couche limite le long d'une paroi plane, à l'exception de la seconde qui exprime la valeur du gradient transversal de pression nécessaire pour contrebalancer l'effet de lat foree centrifuge: cependant, le changement total de pression à travers la couche limite, normalement au fond, est d'ordre o et peut être négligé ${ }^{1}$.

téristique d et d'une vitesse caractéristique $u_{0}$, en posant:

$$
x^{\prime}=\frac{x}{d} \quad y^{\prime}=\mathrm{R}^{1 / 2} \frac{y}{d} \quad u^{\prime}=\frac{u}{u_{0}} \quad v^{\prime}=\mathrm{R}^{1 / 2} \frac{v}{u_{0}}
$$$$
l^{\prime}=\frac{l^{\prime}}{\rho u_{0}{ }^{2}} \quad g^{\prime}=g \frac{d}{u_{0}^{2}} \quad \mathrm{C}^{\prime}=\mathrm{C} d
$$

où $R=\frac{u_{0} d}{v}$, et fait augmenter $R$ indéfiniment. Eu groupant les termes suivant les puissances croissantes de $\frac{1}{\mathrm{R}^{1 / 2}}$, on obtient le système qui régit le mouvement :

$$
\left\{\begin{array}{l}
-\frac{\partial p^{\prime}}{\partial x^{\prime}}=u^{\prime} \frac{\partial u^{\prime}}{\partial x^{\prime}}+v^{\prime} \frac{\partial u^{\prime}}{\partial y^{\prime}}-y^{\prime} \sin i-\frac{\partial^{\prime} u^{\prime}}{\partial y^{\prime 2}}+\frac{1}{\mathrm{R}^{1 / 2}}\left[\mathrm{C}^{\prime} u^{\prime} v^{\prime}-\mathrm{C}^{\prime} y^{\prime} u^{\prime} \frac{\partial u^{\prime}}{\partial x^{\prime}}\right]+\ldots \\
-\frac{\partial p^{\prime}}{\partial y^{\prime}}=\frac{1}{\mathrm{R}^{1 / 2}}\left[u^{\prime} \cos i-\mathrm{C}^{\prime} u^{\prime-2}\right]+\frac{1}{\mathrm{R}}\left[u^{\prime} \frac{\partial v^{\prime}}{\partial x^{\prime}}+v \frac{\partial v^{\prime}}{\partial y^{\prime}}+\mathrm{C}^{\prime} y^{\prime 2} u^{\prime 2}+\frac{\partial^{2} u^{\prime}}{\partial x^{\prime} \partial y^{\prime}}\right]+\ldots \\
\frac{\partial u^{\prime}}{\partial x^{\prime}}+\frac{\partial v^{\prime}}{\partial y^{\prime}}+\frac{1}{\mathrm{R}^{1 / 2}}\left[\mathrm{C}^{\prime} y^{\prime} \frac{\partial v^{\prime}}{\partial y^{\prime}}+\mathrm{C}^{\prime} v^{\prime}\right]=0
\end{array}\right.
$$

La méthode du docteur Goldstern revient done à conserver le terme en $\frac{1}{R^{1 / 2}}$ dans la seconde équation et non pas dans les autres. Mais le résultat final demeure heureusement valable, 
Les trois équations précédentes déterminent le mouvement dans la couche limite, lorsqu'on leur adjoint les conditions aux limites convenables. Soient $u_{1}$ et $p_{1}$ les valeurs de $u$ et $p$ pour $y=\hat{o}$; d'après la remarque précédente, il suffira d'écrive :

$$
\left\{\begin{array}{l}
u \frac{\partial u}{\partial x}+v \frac{\partial u}{\partial y}=-\frac{1}{\rho} \frac{\partial p_{1}}{\partial x}+g \sin i+v \frac{\partial^{2} u}{\partial y^{2}}(4) \\
\frac{\partial u}{\partial x}+\frac{\partial v}{\partial y}=0
\end{array}\right.
$$

avec :

$$
\begin{cases}u=v=0 & \text { pour } y=0 \\ u=u_{1} & \text { pour } y=\delta\end{cases}
$$

Les valeurs de $u_{1}$ et de $p_{1}$ sont fournies par l'étude du mouvement dans la partie extérieure à la couche limite que nous appellerons « noyau »; par hypothèse, les pertes par viscosité y sont négligeables et l'écoulement $y$ est irrotationnel.

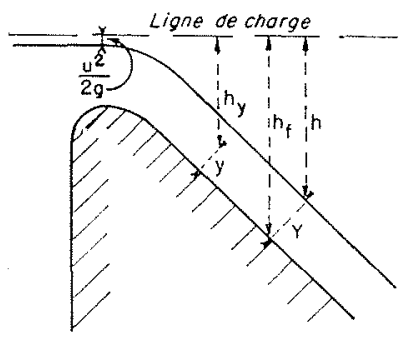

FIG. 2.

Au voisinage de la crête, la couche limite est d'épaisseur faible. Il suffit donc d'étudier l'écoulement d'un fluide parfait sur le fond; on obtient ainsi en chaque point une valeur finie de la vitesse à la paroi qu'on adopte comme valeur de $u_{1}$.

Désignons par $\mathrm{Y}$, fonction de $x$, la profondeur totale de l'écoulement, mesurée normalement au fond, par $h_{f}, h_{y}$ et $h$ les distances verticales entre les points situés à $0, y$ et $Y$ du fond et la ligne de charge définie figure 2. Dans le noyau, la pression et la vitesse sont liées par l'équation de Bernoulti :

$$
\frac{p}{\rho g}+\frac{u^{2}+v^{2}}{2 g}-h_{y}=0
$$

D'où, au fond :

$$
\frac{p_{1}}{\rho g}+\frac{u_{1}^{2}}{2 g}-h_{i}=0
$$

Et à la surface :

$$
\frac{u^{2}+v^{2}}{2 g}-h=0
$$

Soient : $v_{1}, \zeta_{1},\left(\frac{\partial u}{\partial y}\right)_{1}$ les valeurs de $v$, $\zeta$, et $\frac{\partial u}{\partial y}$ pour $y=\delta ;$ comme $\nu_{1}$ est petit, l'équation (1) montre que :

$$
\zeta_{1}=-\left(\frac{\partial u}{\partial y}\right)_{1}-\mathrm{C} u_{1}=0
$$

Done : $\left(\frac{\partial u}{\partial y}\right)_{1}$ est fini.

Plus loin à l'aval, l'épaisseur de la couche limite cesse d'être négligeable. Mais alors, en général, la pente $i$ est forte et la profondeur $Y$ est faible devant la charge $h$ (c'est-à-dire devant la vitesse dans le noyau, d'après la relation de BeRnourci) et devant le rayon de courbure $1 / \mathrm{C}$ du coursier. Nous pourrons appeler cette zone la « zone à faible profondeur $\gg$. Supposons, quitte à le vérifier plus tard, que $\frac{\partial u}{\partial x}$ est fini. Comme $\mathrm{Y}$ est petit, le raisonnement déjà fait à partir de l'équation de continuité montre que $v$ est petit. Si $v$ est une «bonne fonction », comme nous l'avons déjà supposé implicitement, $\frac{\partial v}{\partial x}$ est petit. Or, dans le noyau, par hypothèse, l'écoulement est irrotationnel, donc $\zeta$ est nul; comme CY est supposé petit :

$$
\frac{\partial}{\partial y}[u(1+\mathrm{C} y)] \# \frac{\partial u}{\partial y}=\frac{\partial v}{\partial x}
$$

Donc $\frac{\partial u}{\partial y}$ est petit; $u$ est constant dans le noyau au premier ordre près sur toute normale à la paroi. Les composantes de $-\vec{V} \wedge \vec{\omega}$ $+\overrightarrow{\operatorname{grad}} \frac{1}{2}|\overrightarrow{\mathrm{V}}|^{2}$ ont alors poux partie principale $u \frac{\partial u}{\partial x}$ et $-\mathrm{C} u^{2}$. Comme $\overrightarrow{\operatorname{rot} \vec{V}}=0$, les équations du mouvement dans le noyau loin de la crête se réduisent à :

$$
\left\{\begin{array}{l}
u \begin{array}{l}
\partial u \\
\partial x
\end{array}-g \sin i=-\frac{1}{\rho} \frac{\partial p}{\partial x} \\
-\mathrm{C} u^{2}+g \cos i=-\frac{1}{\rho} \frac{\partial p}{\partial y} \\
\frac{\partial u}{\partial x}+\frac{\partial v}{\partial y}=0
\end{array}\right.
$$


La seconde, compte tenu de la condition $p=0$ pour $y=\mathrm{Y}$ donne :

$$
p=\operatorname{qg}(\mathrm{Y}-y)\left(\cos i--\frac{\mathrm{C} u^{2}}{g}\right)
$$

Au premier ordre près, la pression est constante dans le noyau; au deuxième ordre, elle est pseudo-hydrostatique. Si, en plus des hypothèses faites, la courbure C est faible, la répartition des pressions est la même qu'en régime graduellement varié :

$$
p=\rho g(\mathbf{Y}-y) \cos i
$$

La première équation se réduit en tout cas, au premier ordre près, à :

$$
u \frac{\partial u}{\partial x}=g \sin i
$$

D’après la figure 2 :

$$
\begin{gathered}
h_{i}=h+Y \cos i \\
\frac{d h_{i}}{d x}=\sin i \# \frac{d h}{d x}
\end{gathered}
$$

en remarquant que $\frac{d \mathrm{Y}}{d x}$ est un infiniment petit du premier ordre. D'où :

$$
u=\sqrt{2 g h}
$$

résultat qu'on pourrait aussi déduire de l'écuafion de Bernoula puisque $v$ est petit. De plus :

$$
\frac{\partial u}{\partial x}=\frac{g \sin i}{u}=\frac{g \sin i}{\sqrt{2 g h}}
$$

qui est fini, ainsi que nous le supposions en débutant.

Suivant la théorie classique du mouvement graduellement varié, les conclusions resteraient les mêmes si, au lieu de supposer $Y$ faible, on supposait petite la courbure des filets liquides (C est alors lui-même petit et nous avons vérifié que la répartition des pressions était la même dans les deux cas). En pratique, les applications montrent que les zones où les différentes approximations précédentes sont valables se raccordent sans discontinuité.

\section{II. - Relation intégrale de von Karman.}

Von KARMAN [9] remplace l'équation (3) du mouvement projetée sur $O y$ par l'équation de la quantité de mouvement projetée sur la même di- rection. Suivant une méthode due à PolmauSEN $[10]$, le même résultat peut ètre obtenu en intégrant l'équation (4) par rapport à $y$ entre les limites 0 et $\delta$. Nous poserons :

$$
\nu\left(\frac{\partial u}{\partial y}\right)_{0}=\frac{\tau_{0}}{\rho}=u_{*}^{2}
$$

$\tau_{0}$ désignant le frottement à la paroi.

Nous avons vu que $\left(\frac{\partial u}{\partial y}\right)_{1}$ est fini ou petit; comme v est de l'ordre de $\hat{\delta}^{2}, v\left(\frac{\partial u}{\partial y}\right)_{1}$ est de l'ordre de $\delta^{2}$ au moins et est négligeable. $p_{1}$ étant indépendant de $y$, on obtient :

$$
\begin{aligned}
\ldots\left(\frac{\partial u}{\partial y}\right)_{0} & =\delta\left[\frac{1}{\rho} \frac{\partial p_{1}}{\partial x}-g \sin i\right] \\
& +\int_{0}^{\delta} u \frac{\partial u}{\partial x} \partial y+\int_{0}^{\delta} v \frac{\partial u}{\partial y} d y
\end{aligned}
$$

$\mathrm{Or}:$

$$
\int_{0}^{\delta} v \frac{\partial u}{\partial y} d y=\left[\begin{array}{ll}
u & v
\end{array}\right]_{0}^{\delta}-\int_{0}^{\delta} u \frac{\partial v}{\partial y} d y
$$

D’après l'équation de continuité

$$
\begin{aligned}
& \int_{0}^{\delta} u \frac{\partial v}{\partial y} d y=-\int_{0}^{\delta} u \frac{\partial u}{\partial x} d y \\
& {[v]_{\delta}=\int_{0}^{\delta} \frac{\partial v}{\partial y} d y=-\int_{0}^{\delta} \frac{\partial u}{\partial x} d y}
\end{aligned}
$$

Comme $[v]_{0}=0$ :

$$
\begin{aligned}
u_{*}{ }^{2}=\delta & {\left[-\frac{1}{\circ} \frac{\partial p_{1}}{\partial x}+g \sin i^{-}\right] } \\
& -\int_{0}^{\delta} \frac{\partial u^{2}}{\partial x} d y+u_{1} \int_{0}^{\delta} \frac{\partial u}{\partial x} d y
\end{aligned}
$$

Remarquant que les termes qui contiennent $\frac{d \delta}{d x}$ s'éliminent, et, faisant apparaître $u_{1}-u$ autant de fois que possible, on trouve finalement:

$$
\begin{aligned}
u_{*}^{2} & =\delta\left[-\frac{1}{\rho} \frac{\partial p_{1}}{\partial x}+g \sin i\right]+\frac{d u_{1}}{d x} \int_{0}^{\delta}\left(u_{1}-u\right) d y \\
& +\frac{d}{d x} \int_{0}^{\delta} u\left(u_{1}-u\right) d y-\delta u_{1} \frac{d u_{1}}{d x}
\end{aligned}
$$

Au voisinage de la crête, nous avons vu que $v_{1}$ est petit et que $\left(\frac{\partial u}{\partial y}\right)_{1}$ est fini. La composante 
suivant oy de $-\overrightarrow{\mathrm{V}} \wedge \overrightarrow{(1)}+\overrightarrow{\operatorname{grad}} \frac{1}{2}|\overrightarrow{\mathrm{V}}| 2$ pour le

filet inférieur du noyau se réduit done à $u_{1} \frac{\partial u_{1}}{\partial x}$ et l'équation du mouvement en un point de ce filet s'écrit :

$$
u_{1} \frac{\partial u_{1}}{\partial x}=-\frac{1}{\rho} \frac{\partial p_{1}}{\partial x}+g \sin i
$$

expression identique à celle que nous avons montré être valable plus loin à l'aval. Dans tous les cas, lorsque le noyau existe, (6) se simplifie done en :

$u_{*}^{2}=\frac{d u_{1}}{d x} \int_{0}^{\delta}\left(u_{1}-u\right) d y+\frac{d}{d x} \int_{0}^{\delta} u\left(u_{1}-u\right) d !$

Les équations (6) ou (7) ne sont pas suffisantes pour définir l'écoulement; il est nécessaire de les compléter par des hypothises plus ou moins plausibles touchant la loi de variation de $u$ en fonction de $y$. Cette loi changera suivant la nature du régime, laminaire ou turbulent, dans la couche limite.

\section{CHAPITRE II}

\section{COUCHE LIMITE LAMINAIRE}

Il peut arriver que la couche limite soit laminaire à partir de la crête déversante. Le calcul du mouvement peut alors se faire, dans chaque cas particulier, au moyen de la méthode de PoHLHAUSEN [11] qui donne de bons résultats en écoulement accéléré, comme e'est ici le cas. Elle utilise l'équation (7) en supposant que $\frac{l}{u_{1}}$ est une fonction de $n_{1}=\frac{I}{\delta}$ seulement, représentable par un polynòme du quatrième degré.

Une solution moins exacte mais destince à fixer les ordres de grandeur consiste, suivant par exemple Rouse [12], à représenter $\frac{u}{u_{1}}$ par un polynôme du second degré en $r_{1}$, comme en régime uniforme. Les conditions aux limites sont $\frac{u}{u_{1}}=0$ pour $\eta_{1}=0, \frac{u}{u_{1}}=1$ et $\zeta=0$ pour $r_{1}=1$.

Cette dernière condition se traduit par :

$$
\left(\frac{\partial u}{\partial y}\right)_{1}=\cdots \cdots u_{1}
$$

oll :

au premier ordre près.

$$
\frac{d}{d \eta \eta}\left(\frac{u}{u_{1}}\right)=-\mathrm{C}_{\hat{o}}=0
$$

Le polynôme du second degré cherché est alors :

$$
\frac{u}{u u_{1}}=2 n_{1}--r_{1}^{2}
$$

Et :

$$
\frac{\tau_{0}}{5}=v\left(\frac{\partial u}{\partial !}\right)_{0}=2 \times \frac{u_{1}}{\delta}
$$

Portant ces valeurs dans l'équation (7), il vient :

$$
\text { 30) } y u_{1}=9 u_{1} \frac{d u_{1}}{d x} \delta^{2}+u_{1}{ }^{2} \frac{d \hat{o}^{2}}{d x}
$$

L'écoulement à potentiel faisant connaitre $u_{1}(x)$, cette équation linéaire en ò2 donne numériquement l'évolution de is lorsque les conditions initiales sont fixées.

$\mathrm{Si}$ la couche limite laminaire se prolonge assez loin en aval, nous avons vu qu'alors :

$$
u_{1} \frac{d u_{1}}{d x}=g \sin i
$$

qui est constant si le coursier est plan dans cette zone. Lintégration peut se faire explicitement dans ce cas; si $\delta_{i}$ désigne la valeur de $\delta$ au point pris pour origine où $u_{1}=u_{1}$, on trouve :

$$
\frac{\frac{g \sin i}{3 y} \delta^{2-}-u_{1}}{\frac{g \sin i}{3 y} \delta_{i}^{2}-\mathrm{u}_{1 i}}=\left(\frac{u_{1 i}}{u_{1}}\right)^{3}
$$

Posons $u_{1 i}=\sqrt{2 g h_{i}}$; on observe que $\left(\frac{u_{1 i}}{u_{1}}\right)^{9}$ est 
est une fonction rapidement decroissante de $x$. Elle atteint rapidement par exemple la valeur 0,1 pour :

$$
h=10^{2 / 9} h_{i}=1,67 h_{i}
$$

c'est-à-dire à une distance le long du coursier

$$
x=0,67 h_{i} \sin i
$$

de l'origine. Sauf au voisinage immédiat de co point, on pourra prendre avec une crreur faible :

$$
\delta^{2}=\frac{3 y}{g \sin i} u_{1}
$$

L'épaisseur de la couche limite est donc pratiquement indépendante des conditions amont; c'est là une conclusion générale que nous retrouverons dans le cas de la couche limite turbulente et sur laquelle les auteurs ne semblent pas avoir attiré l'attention.

Le résultat peut être mis sous forme, sans dimension, en fonction du nombre de REynolds :

$$
\begin{gathered}
\mathrm{R}=\frac{u_{1} h}{\nu} \\
\frac{\partial}{h}=\left(\frac{6}{\mathrm{R} \sin i}\right)^{1 / 2}
\end{gathered}
$$

Notons enfin que le débit total q par unité de largeur du coursier est donné par :

$$
q=\int_{0}^{\delta} u d y+u_{1}(\mathrm{Y}-\hat{o})=u_{1}\left(\mathrm{Y} \cdots \frac{\partial}{3}\right)
$$

\section{CHAPITRE III}

\section{COUCHE LIMITE TURBULENTE}

\section{I. - Equation de la quantité de mouvement.}

Ici encore, la relation integrale de Von KARMAN ne peut être utilisée que si l'on précise la loi de variation de $u$ en fonction de $y$. On sait que la théorie et l'expérience montrent que cette loi, étudiée dans le cas des conduites de révolution, doit rester de même forme dans le cas d'un écoulement à deux dimensions. D'après PrandTr [13], elle dépend de la valeur du nombre de REynolds $\mathbf{R}_{*}=\frac{u_{* \varepsilon}}{y}$ (où $\varepsilon$ désigne la « rugosité de NıuRADSE » équivalente de la paroi) et peut se mettre sous la forme générale ${ }^{1}$ :

$\frac{u}{u_{*}}=\frac{1}{k}\left[\log \mathrm{D}-n \log \frac{u_{* \varepsilon}}{y}+\log \frac{y}{\varepsilon}+h\left(\frac{y}{\delta}\right)\right]$

u désignant désormais la vitesse moyenne en un point et $h$ étant une fonction inconnue destinée à tenir compte d'une différence éventuelle entre le cas de la conduite et celui d'une paroi indéfinie. $D$ et $n$ sont des constantes dont la valeur dépend du régime dans la couche limite déterminé par la grandeur de $R_{*}$ (voir tableau $I$ ). $k$ est une

1. Par la suite, nous désignerons par Log les logarithmes népériens ef par log les logarithmes vulgaires. constante universelle dont la valeur est voisine de 0,4 .

On en déduit :

$\frac{u_{1}-u}{u_{*}}=\frac{1}{k}\left[-\log n_{1}+h(1)-h(n)\right]=f\left(r_{1}\right)$

valeur qui, reportée dans l'équation (7), donne :

$$
u_{*}^{2}=\frac{d}{d x}\left[\partial u_{*}\left(u_{1} c_{1}-u_{*} c_{2}\right)\right]+c_{1} \partial u_{*} \frac{d u_{1}}{d x}
$$

où l'on a posé :

$$
c_{1}=\int_{0}^{1} f\left(r_{1}\right) d r_{1} \quad c_{2}=\int_{0}^{1} f^{2}\left(r_{1}\right) d r_{1}
$$

Admettons que $u_{1}$ soit une fonction connue de $x$, calculée suivant la zone que l'on étudie par une des méthodes décrites au chapitre premier, paragraphe I. Posons alors :

$$
k \frac{u_{1}}{u_{*}}=z
$$

$$
\log \mathrm{D}^{\prime}=\log \mathrm{D}+h(1)
$$

d'ò̀ :

$$
\hat{o}=\frac{\varepsilon e^{z}}{\mathrm{I}^{\prime}} \mathrm{R}_{*}{ }^{n}=\frac{\varepsilon^{1+n} k^{n}}{\mathrm{I}^{\prime} \vee^{n}} \frac{e^{z}}{z^{n}} u_{1}^{n}
$$


On se propose de former une relation : $f\left(u_{1}, z, \frac{d u_{1}}{d x}, \frac{d z}{d x}\right)=0$; $u_{1}$ et $\frac{d u_{1}}{d x}$ étant des fonctions connues de $x, f=0$ est une relation différentielle entre $z$ et $x$ : une fois intégrée, elle fournira la valeur de $z(x)$, d'où l'on déduira celle de $\boldsymbol{u}_{*}(\boldsymbol{x})$ qui définit la distribution des vitesses de chaque point.

A cette fin, reportons les valeurs ci-dessus dans (9); il vient :

$\mathrm{A} u_{1}{ }^{2-n}=u_{1}{ }^{2} \frac{e^{z}}{z^{n}}\left[z-\left(1+n+\frac{k c_{2}}{c_{1}}\right)+\frac{k c_{2}}{c_{1}}(2+n) z^{-1}\right] \frac{d z}{d x}+{ }_{z^{n}}^{\rho z} u u_{1} \frac{d u_{1}}{d x}\left[(3+n) z-\frac{k c_{2}}{c_{1}}(2+n)\right]$

avec :

$$
\mathrm{A}=\frac{k^{1-n} \nu^{n} \mathrm{D}^{\prime}}{c_{1} \varepsilon^{1+n}}
$$

C'est la relation cherchée.

Nous discuterons plus loin des conditions aux limites à imposer à cette équation; mais même dans le cas le plus simple où l'on se place loin à l'aval sur un radier plan, il n'est pas possible de l'intégrex. Nous nous contenterons de l'étudier en détail pour $z$ assez grand, ce que nous montrerons être suffisant en pratique.

\section{II. - Valeur des constantes $\mathrm{D}^{\prime}, \mathrm{c}_{1}$ et $\mathrm{c}_{2}$.}

Pour mener les calculs numériques à leur terme, il est nécessaire de connaître $D^{\prime}, c_{1}$ et $c_{2}$. Une première évaluation en est fournie en supposant que la loi de distribution des vitesses dans la conche limite est la même que dans un tuyau en régime uniforme. Alors :

$$
\begin{array}{l|l}
h\left(r_{1}\right)=0 & \mathrm{D}^{\prime}=\mathrm{D} \\
c_{1} & =\frac{1}{k} \# 2,5 \\
l\left(n_{1}\right)=-\frac{1}{k} \log c_{2} & =\frac{2}{k} \# 5
\end{array}
$$

Certaines expériences sembleraient indiquer, à première vue, des valeurs différentes de $D^{\prime}$ et de $c_{1}$ dans la couche limite. En réalité, une discussion détaillée qu'on trouvera à l'annexe I, montre qu'il n'en est rien; pour les valeurs du nombre de Reynolds réalisées, ces résultats ne permettent pas, aux erreurs d'expérience près, de mettre en évidence un écart entre les lois de distribution de la vitesse dans la couche limite en écoulement plan et dans une conduite : nous adopterons donc par la suite les valeurs précédentes et les résultats expérimentaux relatés dans la deuxième partie justifieront encore cette manière de voir.

L'équation (10) s'écrit alors :

$$
\begin{aligned}
\mathrm{A}^{\prime} u_{1}^{2-n} & =u_{1}{ }^{2} \frac{e^{z}}{z^{n}}\left[z-(3+n)+2(2+n) z^{-1}\right] \frac{d z}{d x} \\
+ & \frac{e^{z}}{z^{n}} u_{1} \frac{d u_{1}}{d x}[(3+n) z-2(2+n)]
\end{aligned}
$$

avec :

$$
\mathrm{A}^{\prime}=\frac{k^{2-n v^{n} \mathrm{D}}}{\varepsilon^{1+n}}
$$

Dans le cas général, $u_{1}(x)$ sera déterminé grâce à l'une des méthodes décrites ci-dessus et l'équation (12) pourra être intégrée numériquement.

\section{III. - Etude de la couche limite turbulente sur un radier plan, loin de la crête.}

Dans ce cas, $u_{1} \frac{d u_{1}}{d x}=g \sin i$ et $i$ est constant. L'équation (12) s'écrit alors :

$\frac{d u_{1}}{d z}=\frac{u_{1} e^{z}\left[z^{2}-(3+n) z+2(2+n)\right]}{B z^{1+n} u_{1}{ }^{2-n}-e^{z}\left[(3+n) z^{2}-2(2+n) z\right]}$

où :

$$
\mathrm{B}=\frac{\mathrm{A}^{\prime}}{g \sin i}=\frac{k^{2-n} \nu^{n} \mathrm{D}}{\varepsilon^{1+n} g \sin i}
$$

L'étude détaillée des courbes intégrales pour les différentes valeurs de $n$ est donnée à l'annexe II; on constate en particulier que toutes les intégrales admettent une solution asymptotique commune dont elles se rapprochent très rapidement; nous avons déjà rencontré les mêmes circonstances au chapitre II, dans le cas de la couche limite laminaire, et à l'annexe I dans le cas de la couche limite turbulente le long d'une plaque plane lisse plongée dans un courant uniforme. M. Comolet [14] avait démontré le fait pour la couche limite dans un convergent de révolution; il est d'ailleurs possible de transposer son mode de calcul au problème qui nous occupe (annexe III). Il semble donc qu'il y ait là une propriété très générale; dans une publication ultérieure, nous nous attacherons à préciser ce point. Le problème peut être énoneé mathémati- 
quement comme suit : étant donné une équation différentielle :

$$
\frac{d u_{1}}{d z}=\frac{f\left(u_{1} z\right)}{g\left(u_{1} z\right)}
$$

possédant à l'infini une singularité isolée, reconnaittre les classes de fonctions f et $g$ (supposées suffisamment régulières) pour lesquelles le fait mentionné a lieu. Une fois ce résultat obtenu, il reste à reconnaitre que les lois physiques proposées pour la distribution des pitesses conduisent à des fonctions f et g entrant dans les classes mises ainsi en évidence.

Dans le cas présent, quelles que soient les conditions initiales, on peut alors adopter cette solution asymptotique comme approximation de la solution exacte, suffisamment loin en aval du point initial. Son expression, à des termes en $z^{-3}$ près, est :

$u_{1}^{2-n}=\frac{5}{\mathrm{~B}} z^{1-n} e^{\approx}\left[1+a_{1} z^{-1}+a_{2} z^{-2}\right]$

où :

$$
\left\{\begin{array}{l}
a_{1}=-\frac{2}{5}(6-n) \\
a_{2}=\frac{4}{25}(2-n)(7-2 n)
\end{array}\right.
$$

Le tableau I résume les valeurs des constantes, suivant la nature du régime, fonction de la valeur de :

$$
\mathrm{R}_{*}=\frac{u_{*} \varepsilon}{\nu}=k \frac{u_{1}}{\nu} \frac{\varepsilon}{z}
$$

\begin{tabular}{|c|c|c|c|c|c|}
\hline RígrMe & VALEUR DE $R_{*}$ & $n$ & D & $a_{1}$ & $a_{2}$ \\
\hline Lisse L.. & $R_{*}<5,08$ & -1 & 9,01 & $-2,8$ & 4,32 \\
\hline De transition $\mathrm{T}_{1} \ldots$ & $5,08<R_{*}<14,1$ & 0 & 46,04 & $-2,4$ & 2,24 \\
\hline De transition $\mathrm{T}_{2}$. & $14,1<\mathrm{R}_{*}<70,8$ & 0,282 & 99,17 & $-2,29$ & 1,85 \\
\hline Rugueux $\mathrm{R}$. & $70,8<\mathrm{R}_{*}$ & 0 & 29,66 & $-2,4$ & 2,24 \\
\hline
\end{tabular}

TABLEAU I

L'équation (14) fournit la solution du problème. On peut la mettre sous une autre forme en faisant apparaître $\delta$ :

$$
u_{1}^{2}=\frac{5}{k^{2}} g \circ \sin i\left[z+a_{1}+a_{2} z-1\right]
$$

ou encore sous forme sans dimension :

$$
\frac{2}{5} \frac{k^{2}}{\sin i} \frac{h}{\delta}=z+a_{1}+a_{2} z-1
$$

De même, en faisant apparaître

et $\mathrm{R}_{*}$ :

$$
\mathrm{R}=\frac{u_{1} h}{\nu}=\frac{u_{1}^{3}}{2 g^{\nu}}
$$

$$
\mathrm{R}=\frac{5 \sin i}{2 k^{3} \mathrm{D}} \mathrm{R}_{*}{ }^{1+n} e^{z}\left(z^{2}+a_{1} z+a_{2}\right)
$$

A cause de la présence du facteur $e^{z}$, l'équation (14) montre que $z$ varie peu pour d'assez grandes variations de $u_{1}$. On a donc sensiblement, d'après (16) :

$$
\frac{d \grave{\delta}}{d h}=\frac{d \hat{o}}{d x} \frac{1}{\sin i}=\frac{2}{5} \frac{k^{2}}{\sin i} \frac{1}{z+a_{1}+a_{2} z^{-1}}
$$

Pour $z=8$, ce qui est une valeur moyenne, ainsi que nous le verrons plus loin :

$$
\frac{d \delta}{d x} \# 0,008
$$

La divergence de la couche limite est très faible, de l'ordre de $0^{\circ} 30^{\prime}$. On s'explique bien ainsi que la distribution des vitesses puisse y être voisine de celle du régime uniforme. 
On trouve alors:

- en régime $\mathrm{L}_{\ldots} \ldots \mathrm{R}_{*}<5,0 \mathrm{~S}$

$$
z>6,75
$$

- en régime $\mathrm{r}_{1} \ldots \mathrm{R}_{*}<1,1$

$z>5,9$

- en régime $\mathrm{T}_{2} \ldots \mathrm{R}_{*}<70,8$

$z>4,1$

- en régime $\mathrm{R}$, si $\mathrm{R}_{*}<100$

$z>3,84$

En se plaçant dans les conditions les plus défavorables, $z$ est encore grand devant l'unite pour $\mathbf{R}_{*}<100$. Sauf pour les parois très rugueuses, cela justifie le fait d'avoir utilisé la solution (14).

Par conséquent, la seule connaissance du nombre de REyolds de transition fixera la position du point de transition, la forme de la couche limite étant définie en aval. Réciproquement, imposer au point de transition une relation physique entre la couche laminaire ef la couche turbulente revient a fixer ce nombre de RFynoms. L'indépendance de la forme de la couche limite vis-à-vis des conditions amont étant un fait très général d'après ce que nous avons vu précédemment, éest même peut-être là la raison profonde de la fixité constatée du nombre de RExNoLds au point de transition. Il semble que cette remarque n'ait pas été explicitée antéricurement.

Une autre difficulté se présente lorsque lalimentation n'est pas parfaitement calme ou lorsque la rugosité (répartie on localisée) est forte au voisinage de la crete : Ia couche limite peut devenir turbulente dans une zone oì les calculs précédents ne sont pas valables. L'expression de $u_{1}(x)$ n'étant pas connue, il n'est pas possible de faire une théorie générale; cependant, de tout ce qui précède, il semble bien résulter que l'évolution de la couche limite dépendra peu des conditions initiales que nous Jui imposerons pour le calcul. En faisant par exemple l'hypothese raisonnable $\delta_{i}=\varepsilon$, on trouve, d'après l'équation (10), $z_{i}$, $\delta_{i}$ et $u_{1 i}$ désignant les valeurs initiales de $z, \delta$ et $u_{1}$ :

-.- en régime L... $\hat{o}_{i}=0$

$z_{i}=0$

con régime $\mathrm{T}_{1} \ldots \frac{e^{*}}{\mathrm{D}}=-1 \quad z_{i}=3,84$

- en régime $\mathrm{T}_{2} \ldots \frac{k^{n}}{\mathrm{D}} \frac{e^{z i}}{z_{i}^{n}}\left(\frac{u_{1 i} \varepsilon}{\nu}\right)^{n}=1 z_{i}=3,39$

- en régime $\mathrm{R} \ldots \frac{e^{z_{i}}}{\mathrm{D}}=1 \quad z_{i}=3,39$

\section{VI. - Similitude de la position des points critiques.}

II s'agit dans ce paragraphe de comparer la position des points criliques sur un prototype et sur un modèle en similitude de Frovde au $1 / \mu$.
Nous désignerons les grandeurs se rapportant au prototype par des lettres accentuées.

Il est assez délicat de faire un exposé général de cette similitude, car la nature des écoulements au point critique dépend des valeurs des nombres de REYNolds $\mathrm{R}_{*}$ et $\mathrm{R}_{*}^{\prime}$; or :

$$
\frac{\mathrm{R}_{*}^{\prime}}{\mathrm{R}_{*}}=\frac{u_{1}^{\prime}}{u_{1}} \frac{\varepsilon^{\prime}}{\varepsilon} \frac{z}{z^{\prime}}
$$

en supposant que $y==y^{\prime}$.

A priori, on peut dire que $z$ est assez peu diflérent de $z^{\prime}$ puisque nous avons vu que $z$ varie peu avec $q$; $u^{\prime}{ }_{1}$ serait voisin de $y^{1 / 2} u_{1}$ si le modèle suivait la loi de similitude de Frovdr. Si $\varepsilon^{\prime}$ est voisin de $\varepsilon, R_{*}^{\prime}$ sera done voisin de e $^{1 / 2} R_{*}$ Le régime d'écoulement du prototype sera souvent différent de celui du modèle, et en général plus rugueux. Suivant les valeurs de y. et de $\hat{\varepsilon}^{\prime} / \varepsilon$, toutes les combinaisons de régime deux à deux sont possibles.

Supposons tout d'abord le modèle et le prototype en régime lisse. L'équation (26) permet d'écrire :

$$
\begin{aligned}
& 0,4803\left(z^{\prime}-z\right)=\log \frac{q^{\prime}}{q}=\frac{3}{2} \log \mu \\
& z^{\prime}-z=3,13 \log \omega=\mu_{\mathrm{I}_{2}}
\end{aligned}
$$

D’après (17) et (24):

$$
\begin{aligned}
& \frac{h^{\prime}}{h}=\varphi\left[\frac{\left(z^{\prime 2}+a_{1} z^{\prime}+a_{2}\right)(z-1)}{\left(z^{2}+a_{1} z+a_{2}\right)\left(z^{\prime}-1\right)}\right]^{2 / 3} \\
& \frac{h^{\prime}}{h}=\mu\left[1+\frac{2}{3} \frac{v_{-}}{z}+\ldots\right]
\end{aligned}
$$

$h^{\prime}$ sur le prototype sera toujours plus grand que u.h, mais la différence sera d'autant moins grande que :

1" $\mu_{\mathrm{I}}$ sera plus petit, donc $\mu$ plus voisin de 1 , ce qui est évident;

$2^{\prime \prime} z$ sera plus grand, c'est-à-dire que $h$ sera plus grand au point critique.

L'équation (16) donne alors :

$\mathrm{Y}_{c}^{\prime}=\frac{h^{\prime}}{h} \frac{z^{\prime}\left(z^{2}+a_{1} z+a_{2}\right)}{z\left(z^{\prime 2}+a_{1} z^{\prime}+a_{2}\right)}=\mu\left(1-\frac{1}{3} \frac{\mu_{L}}{z}+\cdots\right)$

$Y_{c}$ est donc inférieur à $\mu Y_{c}$. Remarquons encore que :

$$
\frac{u_{1}^{\prime}}{u_{1}}=\sqrt{\frac{h^{\prime}}{h}}=u^{1 / 2}\left[1+\frac{1}{3} \frac{u_{\cdot J_{-}}}{z}+\ldots\right]
$$


L'utilisation pratique de la formule (14) est assez mal commode. On peut encore l'écrire :

$$
\begin{aligned}
z \log e+\log \left(z^{1-n}\right. & \left.+a_{1} z^{n}+a_{2} z^{-1-n}\right) \\
& =\log \frac{k^{2-n} \mathrm{D}}{5}\left(\frac{y}{u_{1} \varepsilon}\right)^{n} \frac{u_{1}{ }^{2}}{\varepsilon g \sin i}
\end{aligned}
$$

La variation du premier membre est presque linéaire dans un large domaine de variation de $z$. Pour $7<z<15$ par exemple, on pourra dégrossir les calculs par les formules d'interpolation suivantes :

\section{Régime I. :}

$$
0,5274 z=-1,8524+\log \frac{u_{1}^{3}}{\vee g \sin i}
$$

$\frac{\Delta z}{z}$ maximum $=0,9 \%$ pour $z=-7$

If faut cependant prendre garde que :

$$
\frac{\Delta e^{2}}{e^{2}}=z\left(\frac{\Delta z}{z}\right)=6,3 \%
$$

On voit pourquoi la formule (18) doit être utilisée avec précaution, en ce qui concerne par exemple le calcul de o par la formule (10). I] faudra de préférence appliquer l'équation (16).

RÉgine ' $\mathrm{l}_{\mathrm{T}}$ :

$0,4860 z=-0,1848+\log \frac{u_{1}^{2}}{\varepsilon g \sin i}$

$\frac{\Delta z}{z}$ maximum $=0,7 \%$ pour $z=-7$

RÉGINE 'T.2 :

$0,4741 z=0,4152+\log \left(\frac{\nu}{u_{1} \varepsilon}\right)^{0,2 s 2} \frac{u_{1} z}{\varepsilon g \sin i}$

$\frac{\Delta z}{z}$ maximum $=0,57 \%$ pour $z=-7$

RÉgime R :

$0,4860 z=-0,3758+\log \frac{u_{1}{ }^{2}}{\varepsilon g \sin i}$

$\frac{\Delta z}{z}$ maximum $=0,7 \%$ pour $z=7$

Remarquons enfin que le débit $q$ par unité de largeur est donné par :

$q=\int_{0}^{\delta} u d y+u_{1}(\mathrm{Y}-\hat{\delta})=u_{1} \mathrm{Y}-\int_{0}^{\delta}\left(u_{1}-u\right) d y$

car on commet une erreur tres faible en suppo- sant que la valeur de $u$ est donnée par une loi logarithmique jusqu'à la paroi [15]. D'où :

$$
\gamma=\mathbf{U Y}=u_{1}\left[\mathrm{Y}-\frac{k c_{1}}{z} \delta^{-}\right]=n_{1}\left[\mathrm{Y}-\frac{\partial}{z}\right]
$$

si U désigne la vitesse noyenne.

\section{IV. - Position du point critique.}

Nous aborderons maintenant l'étude de la notion de point critique, qui constitue peut-être notre apport le plus original à la théorie. En ce point, la couche limite atteint la surface libre et l'aspect même de l'écoulement se trouve profondément altéré. Nous nous proposons la détermination théorique de la position du point critique, et il semble opportun de signaler dès maintenant que la concordance de nos prévisions avec l'expérience se trouve être des plus satisfaisantes. Pour des débits très faibles, le point critique pourrait théoriquement être situé dans la zone de la crête; néanmoins, les applications traitées dans la seconde partie montrent que, en pratique, il se trouvera toujours situé dans la zone à faible profondeur; les calculs qui suivent seront faits dans cette hypothèse.

Ecrivons que la profondeur $Y_{c}$ de l'écoulement au point critique est égale à $\delta$; les valeurs de $u_{1}, Y, h$ et $z$ sont alors définies par le système :

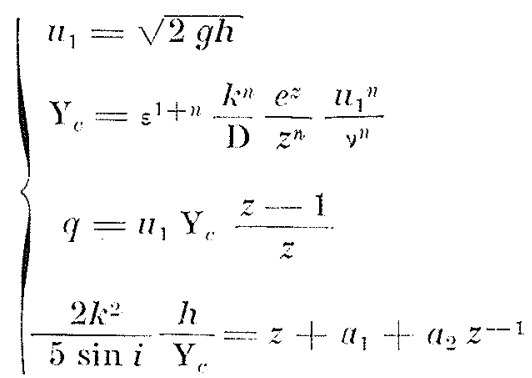

Une ou deux des trois dernieres relations peut d'ailleurs etre remplacéc par une de leurs combinaisons telle que (14) ou (17).

De (22) et (23), on tire aussi :

$$
\frac{q}{\nu}=\frac{1}{k \mathrm{D}} \mathrm{R}_{*}{ }^{1+n} e^{z}(z-1)
$$

(qui détermine $z$ en fonction de $q$ lorsque $n=-1)$.

Liéquation qui lie $z$ au point critique à $q$ s'obtient en éliminant $u_{1}$ et $R_{*}$ entre (24), (17) et (14) :

$$
e^{3 z} \frac{\left(z^{2}+a_{1} z+a_{2}\right)^{1+n}}{z^{3(1+n)}(z-1) 2-n}=\frac{k^{2}-n \mathrm{D}^{3}}{5^{1+n}}\left(\frac{q}{\nu}\right)^{2-n}\left(\frac{\nu^{2}}{\left.\varepsilon^{3} g \sin i\right)}\right)^{1+n}
$$


Le premier membre de cette équation est équivalent pour $z$ grand à $e^{3 z} z^{1-2 n}$ avec $1-2 n>0$; $z$ augmente donc avec $q$, mais varie peu dans un large domaine de variation de $q$ car $e^{3 *}$ croît très vite avec $z$. Dans ces conditions, (16) montre que $\frac{h}{\mathrm{Y}_{c}}$ augmente lentement avec $q$; de même (14) montre que $u_{1}$, done $h$, augmentent lorsque $q$ croît; le point critique se déplace vers l'aval lorsqu'on augmente le débit sur un coursier donné. On explique ainsi le fait, à première vue paradoxal, que l'eau blanche puisse apparaître sur un ouvrage à débit donné et ne pas apparaître lorsque le débit est augmenté, quoique les vitesses réalisées soient alors plus grandes. Il suffit pour cela que le point critique ne soit plus situé sur le déversoir dans le second cas.

La formule (25) étant peu pratique à manier, on peut, comme précédemment, chercher des formules d'interpolation pour $7<z<15$.

Régrme I - (25) s'écrit :

$e^{\approx}(z-1)=\frac{k \mathrm{D} q}{y}$

remplacé par :

$0,4803 z=0,0814+\log \frac{q}{\nu}$

$\frac{\Delta z}{z} \operatorname{maximum}=0,57 \%$ pour $z=7$

RÉgIME $\mathrm{T}_{1}-(25)$ s'écrit :

$e^{3 z} \frac{z^{2}-2,4 z+2,24}{z^{3}}(z-1)^{2}=\frac{k^{2} \mathrm{D}^{3}}{5}\left(-\frac{q}{\nu}\right)^{2} \frac{\nu^{2}}{\varepsilon^{3} g \sin i}$

remplacé par :

$1,362 z=3,307+\log \left(\frac{q}{\nu}\right)^{2} \frac{\nu^{2}}{\varepsilon^{3} g \sin i}$

$\frac{\Delta z}{z}$ maximum $=0,3 \%$ pour $z=7$

RÉGIME $T_{2}-(25)$ s'écrit :

$e^{3 z} \frac{\left(z^{2}-2,29 z+1,85\right)^{1,282}}{z^{3,846}}(z-1)^{1,718}$

$$
=\frac{k^{1,718} \mathrm{D}^{3}}{5^{1,282}}\left(\frac{q}{\nu}\right)^{1,718}\left(\frac{\nu^{2}}{\varepsilon^{3} g \sin i}\right)^{1,282}
$$

remplacé par :

$1,342 z=4,605+\log \left(\frac{q}{y}\right)^{1, \pi 18}\left(\frac{v^{2}}{\varepsilon^{3} g \sin i}\right)^{1,282}$

$\frac{\Delta z}{z}$ maximum $=0,26 \%$ pour $z=7$

Régime R - (25) s'écrit :

$e^{3 z} \frac{z^{2}-2,4 z+2,24}{z^{3}}(z-1)^{2}=\frac{k^{2} \mathrm{D}^{3}}{5}\left(\frac{q}{v}\right)^{2} \frac{\nu^{2}}{\varepsilon^{3} g \sin i}$ remplacé par :

$1,362 z==2,7340+\log \left(\frac{q}{v}\right)^{2} \frac{v^{2}}{\varepsilon^{3} g \sin i}$

$\frac{\Delta z}{z}$ maximum $=0,3 \%$ pour $z=7$

\section{V. - Discussion des conditions initiales de la couche limite turbulente.}

En général, le régime, laminaire à partir de la crête, devient turbulent à l'aval; il faudra tâtonner pour savoir quel est le régime qui s'établit alors et, pour cela, examiner la manière dont se fait le passage de la zone laminaire à la zone turbulente.

Cette question est complexe et encore mal éclaircie. La transition a lieu sur une certaine longueur, mais comme on ne connaît pas les lois du mouvement dans cette zone, on est amené, pour la commodité du calcul, à supposer qu'elle se produit instantanément en un point, dit point de transition. Dans le cas d'une plaque plane plongée dans un courant de vitesse uniforme $u_{1}$, la distance $x_{i}$ du point de transition au bord d'attaque est caractérisée par la valeur du nombre de Rexnolos $\mathrm{R}_{t}=\frac{u_{1} x_{t}}{y}$, qui est comprise entre $10^{5}$ et $10^{6}[16],[17]$.

Dans l'étude ci-dessus, nous avons rencontré, comme nombre de RexNolds fixant la position d'un point sur le coursier, l'expression $\mathrm{R}=\frac{u_{1} h}{\nu}$. D'ailleurs, si le radier devient plan en un point où la charge $h_{0}$ est faible :

$$
h=h_{0}+x \sin i \# x \sin i
$$

et

$$
\mathrm{R}=\mathrm{R}_{t} \sin i \text {. }
$$

L'expérience seule permettrait de fixer quelle est l'expression du nombre de Rernolos qui détermine la position du point critique et quelle est sa valeur.

Ceci étant précisé, il resterait à donner la relation entre la couche laminaire et la couche turbulente au point de transition, autrement dit la condition initiale à imposer à la couche turbulente. La multiplicité même des hypothèses proposées montre qu'aucune d'elles n'est très satisfaisante [18]. Très heureusement, on peut voir que, dans la plupart des cas, $z$ sera grand devant l'unité au point de transition, c'est-à-dire que la forme de la couche limite en aval ne dépendra pratiquement pas de cette condition initiale.

Pour le montrer, utilisons l'équation (17). On obtiendra certainement des valeurs trop faibles de $z$ au point critique en $y$ faisant $\mathrm{R}=10^{5} \sin i$. 
et que :

$$
\left.\frac{R_{*}^{\prime}}{R_{*}}=\frac{u_{1}^{\prime}}{u_{1}} \frac{z}{z^{\prime}} \frac{\varepsilon^{\prime}}{\varepsilon}=\mu^{1 / 2}\left(1-\frac{2}{3} \frac{y_{\mathrm{L}}}{z}+\ldots\right)\right)_{\varepsilon}^{\varepsilon^{\prime}}
$$

Si $R_{*}^{\prime}$ tiré de cette formule est inférieur à 5,08 , le régime sur le prototype est effectivement lisse. Sinon, considérons un prototype de rugosité fictive $s_{f}^{\prime}$ telle que la condition ci-dessus donne juste $\mathrm{R}_{*}^{\prime}=5,08$. La rugosité réelle $\varepsilon^{\prime}$ sera supérieure à $\varepsilon_{j}^{\prime}$ et la formule (27) montre que $z^{\prime}$ réel sera inf'́rieur à $z^{\prime}$ fictif, ce qui, si l'on veut, peut se traduire par une diminution de $\mu_{x} \cdot \frac{h^{\prime}}{h}$ réel sera inférieur à $\frac{h^{\prime}}{h}$ fictif et tendra done à se rapprocher de la valeur p.

Examinons encore le cas où modèle et prototype sont en régime rugueux et posons :

$$
\frac{\varepsilon^{\prime}}{\varepsilon}=\eta_{1}
$$

Sauf précautions spéciales, $\mu_{1}$ sera différent de et lui sera en général inférieur. De (29) on tire :

$$
\begin{aligned}
& 1,363\left(z^{\prime}-z\right)=\log \frac{q^{\prime 2}}{q^{2}} \frac{\varepsilon^{3}}{\varepsilon^{\prime 3}}=3 \log \frac{y_{0}}{y_{1}} \\
& z^{\prime}-z=2,2 \log \frac{\mu_{0}}{u_{1}}=\mu_{3}
\end{aligned}
$$

Comme ci-dessiss, on peut écrire :

$$
\begin{aligned}
& \frac{h^{\prime}}{h}-=\left[1+\frac{2}{3} \frac{\mu_{\mathrm{R}}}{z}+\ldots\right] \\
& \frac{\mathrm{Y}^{\prime} \mathrm{C}}{\mathrm{Y} c}=u \cdot\left[1-\frac{1}{3} \frac{u_{\mathrm{R}}}{z}+\ldots\right] \\
& \left.\frac{\mathrm{R}_{*}^{\prime}}{\mathrm{R}_{*}}=\mu, \mu-\frac{2}{3} \frac{\mu_{\mathrm{R}}}{z}+\ldots\right]
\end{aligned}
$$

La similitude de Froude sera réalisée dans le seul cas où $y=p_{1}$. On aura alors :

$$
\frac{R^{\prime} *}{R_{*}}=\mu^{3 / 2}
$$

Lorsque $u_{1}$ sera inférieur à $u$, les conciusions précédentes restent valables :

$$
\begin{gathered}
h^{\prime}>\mu h \\
\mathrm{Y}^{\prime} c<u \mathrm{Y} c
\end{gathered}
$$

Il serait vain d'écrire les formules pour toutes les combinaisons possibles de régimes deux à deux, car les conclusions dépendent entièrement des valeurs relatives des rugosités du modèle el du prototype. Il faudra faire le calcul dans chaque cas d'espèce. Les calculs précédents montrent cependant que la similitude de Frovde est à peu près vérifice lorsque "s. n'est pas trop grand. Et c'est la seule conclusion générale à laquelle aboutit l'étude précédente.

\section{VII. - Evolution du mouvement en aval $\mathrm{du}$ point critique.}

Au point critique, la turbulence est généralisée dans toute la hauteur de l'écoulement. Si l'eau blanche ne se produit pas, la répartition des vitesses va continuer à se modifier vers l'aval pour tendre vers le régime uniforme lorsque la paroi est plane; nous nous proposons d'étudier cette évolution.

Dans le cas lẹ plus fréquent où le point critique est situé dans la zone à faible profondeur. les hypothèses de la couche limite seront applicables en aval à la hauteur totale de l'écoulement. Comme il n'existe désormais plus de noyau, la relation intégrale de von Karman doit être utilisée sous la forme (6) où $p_{1}=0$ et $\mathrm{Y}=\delta$. Si la loi logarithmique de distribution des vitesses continue à être vérifiée, on trouve ainsi :

$$
\begin{aligned}
u_{*}{ }^{2}=g \mathrm{Y} \sin i \cdots \mathrm{Y}\left(u_{1}-u_{*} c_{1}\right) \frac{d u_{1}}{d x} \\
+\frac{d}{d x}\left[\mathrm{Y} u_{*}\left(u_{1} c_{1}-u_{*} c_{2}\right)\right]
\end{aligned}
$$

Si $i$ est constant, on voit bien qu'un régime permanent s'établit et que $u_{*}$ tend vers sa valeur normale $u_{*_{n}}=\sqrt{g Y_{n} \sin i}$, correspondant à la profondeur $Y_{n}$ de l'écoulement uniforme.

Désormais, les couches supérieures ne sont plus en régime uniformément accéléré; la relation nécessaire pour définir $n_{1}$ est alors l'équation (24) qui exprime l'invariance du débit unitaire. Par différenciation logarithmique, on en déduit :

$$
\frac{d z}{d x}=-\frac{1}{u_{*}}(z-1)(1+n) \frac{d u_{*}}{d x}
$$

Ce calcul n'est pas valable en régime lisse lorsque $n=-1$; mais (24) montre qu'alors constant en aval du point critique, par c quent (31) est encore vérifié. 
Reportant dans (30) :

$$
u_{*}{ }^{\prime \prime}=\frac{k q}{z-1}\left\{\left(\left(g \sin i-\frac{1}{l^{2}{ }^{2}} u_{*} \frac{d u_{*}}{d x}\left[z^{2}-2 z+2-(1+n)(z-2)\right]\right)\right\}\right.
$$

Eliminons $u_{*}$ entre (32) et (24); il vient pour $n \neq \neq-1$ :

$$
\left[k^{2-n} \mathrm{D}^{3} \frac{q^{2-n} \mathrm{y}^{3 n}}{\varepsilon^{3(1+n)}} \frac{1}{e^{3 z}(z-1)^{2-n}}\right]^{\frac{1}{1+n}}=g \sin i+\frac{1}{k^{2}}\left[\frac{l i \mathrm{D}}{e^{z}(z-1)} \frac{q^{y^{n}}}{z^{1+n}}\right]^{\frac{2}{1+n} z\left[z^{2}-2 z+2-(1+n)(z-2)\right]} \frac{d z}{d x}(3
$$

qui définit l'évolution de $z$ en fonction de $x$.

On voit que la valeur de $z$ pour le régime uniforme vérifie :

$$
k^{2-n} \mathrm{D} \cdot\left(\frac{q}{y}\right)^{2-n}\left(\frac{v^{2}}{\varepsilon^{3} g \sin i}\right)^{1+n}-e^{3 z}(z-1)^{2-n}=0
$$

ainsi qu'on aurait pu l'établir directement, quel que soit $n$.

Le sens de variation de $z$ en aval du point eritique dépend du signe du premier membre de l'équation (33) au point critique; s'il est négatif, $z$ décroìt, donc $u_{*}$ croît; d'après (25), il suffit pour cela qu'au point critique :

$$
z>5\left(1+a_{1} z^{-1}+a_{2} z^{-2}\right)
$$

La discussion du paragraphe $Y$ montre gue cette condition sera remplie en régime $x$ et ${ } T_{1}$ et le sera aussi en général dans les régimes $\mathrm{T}_{2}$ et $R$.

L'équation différentielle du mouvement peut aussi s'exprimer en fonction de $Y$. Prenons pour cela la dérivé logarithmique de :

$$
\begin{gathered}
q=\frac{u_{*} Y}{k}(z-1) \\
\frac{1}{u_{*}} \frac{d u_{*}}{d x}+\frac{1}{Y} \frac{d Y}{d x}+\frac{1}{z-1} \frac{d z}{d x}=0
\end{gathered}
$$

D'après (31) on trouve, quel que soit $n$ :

$$
\frac{1}{\mathrm{Y}} \frac{d \mathrm{Y}}{d x}+\frac{1}{u_{*}} \frac{z-(1+n)}{z} \frac{d u_{*}}{d x}=0
$$

Reportons cette expression dans (32) :

$$
\frac{q^{2}}{g} \frac{k^{2}}{(z-1)^{2}} \frac{1}{Y^{3}}-\sin i=\frac{q^{2}}{g} \frac{1}{Y^{3}} \frac{d Y}{d x}\left[1+z^{2}+\ldots\right]
$$

En régime lisse, $z$ est constant en aval du point critique el cette équation définit l'évolution du monvement. Dans tout autre regime, z est variable; cependant la comparaison de (33) et de (25) montre, à cause de la prépondérance du terme en $e^{3 z}$, que la valeur de $z$ varie peu entre le point critique et le régime normal. On pourra donc diviser l'intervalle d'intégration en sections où $z$ sera sensiblement constant, et utiliser encore l'équation (36).

D'après (16) et (23), au point critique :

$\frac{q^{2}}{g} \frac{k^{2}}{(z-1)^{2}} \frac{1}{\mathrm{Y}^{3}}=\sin i \frac{5\left(1+a_{1} z^{-1}+a_{2} z^{-2}\right)}{z}$ et le premier membre de (36) est négatif si (34) esl vérifié all point critique.

En conclusion, lorsqu'il en est ainsi, en aval du point critique, $u_{*}$ croît alors que $\mathrm{Y}$ décroit ainsi que $z$.

Posons, suivant l'habitude :

$$
\frac{q^{2}}{g}=\mathrm{Y}_{c r^{\prime}}: \quad \tau_{\theta}=-\frac{g}{\mathrm{C}^{2}}-\mathrm{U}^{2}
$$

e'est-à-dire ${ }^{1}$ :

$$
\frac{g}{C^{2}}=\frac{k k^{2}}{(z-1)^{2}}
$$

L'équation (36) s'écrit avec ces notations : $\sin i-\frac{\mathrm{Y}_{c r^{3}}}{\mathrm{Y}^{3}} \frac{g}{\mathrm{C}^{2}}=\frac{d \mathrm{Y}}{d x}\left[-\frac{\mathrm{Y}_{c r^{3}}}{\mathrm{Y}^{3}}\left(1+z^{-2}+\ldots\right)\right]$ $z$ étant grand, $1+z^{-2}$ est peu différent de l'unité et on reconnait l'équation habituelle du mouvement graduellement varié dans un canal très large, lorsque le mouvement est fortement torrentiel ( $i$ remplacé par $\sin i, \cos i$ négigé devant $\left.\frac{\mathrm{Y}_{r, \cdot 3}}{\mathrm{Y}^{3}}\right)$. On pouvait s'y attendre puisque, pour obtenir cette équation, on fait également l'hypothèse de la répartition hydrostatique des pressions et que l'on peut appliquer ensuite le théorème des quantités de mouvement. On est cependant amené à faire certaines hypothèses sur la nature de la perte de charge et à négliger l'inégale répartition des ritesses dans la section; notre calcul, plus détaillé, montre que ce mode de raisonnement est tout à fait justifié.

Intégrons (36) en supposant $z$ constant. On voit que $Y$ tend vers la profondeur normale définie par :

$$
Y_{n}:=\frac{Y_{r r^{3}} g}{C^{2} \sin i}=\frac{q^{2}}{C^{2} \sin i}
$$

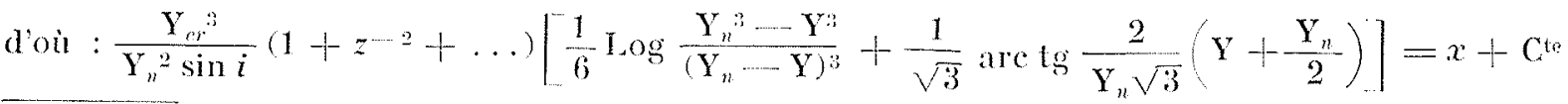

1. Aucume confusion n'est possible cutre le coefficient C de Chezy introduit ici et la combure C $(x)$ du coursier. 
Nous avons vu que $z$, donc $Y$, varient peu entre le point critique et le régime normal; cherchons donc une formule pratique en posant :

$$
\mathrm{Y}=\mathrm{Y}_{n}(1+x)
$$

Développant dans (36) et intégrant, il vient :

$$
\begin{aligned}
\mathrm{F}(\mathrm{x}) & =-0,7675 \log x+\frac{x}{3}-\frac{x^{2}}{9}+\frac{x^{3}}{27}-\frac{x^{4}}{108} \\
& +\ldots=x \frac{\mathrm{Y}_{n}^{2} \sin i}{\mathrm{Y}_{0} ;\left(1+z^{2}+\ldots\right)}+\mathrm{K}
\end{aligned}
$$

où K est la constante d'intégration dépendant des conditions initiales. La courbe représentative de $F(x)$ est donnée figure 3 .

\section{REMARQUES :}

$1^{\prime \prime} \quad l_{*}$ croissant en régime lisse à partir du point critique, si le régime uniforme est lisse, on est certain a priori que le régime est lisse au point critique.

2" En régime lisse, $z$ est constant en aval du point critique. Pour les autres régimes, sa valeur limite est définie par (33). Les autres grandeurs caractéristiques du régime normal sont alors données par :

$$
\begin{aligned}
& u_{*}=\left[\frac{k q g \sin i}{z-1}\right]^{1 / 3} \\
& \mathrm{Y}_{n}=\frac{u_{*}^{2}}{g \sin i} \\
& u_{1}=\frac{u_{*}}{k}+\frac{q}{\mathrm{Y}_{n}}
\end{aligned}
$$

3" On peut présenter sous forme synthétique l'ensemble de l'étude nécessaire pour décrire completement la mise en régime de l'eau sur un déversoir suivi d'un radier plan à forte pente.

I. - ZONE DE LA CRÈTE. - L'étude classique par la mécanique des fluides parfaits fournit la valeur de la vitesse à la paroi $u_{1}(x)$. Les équations (8) oụ (12) définissent respectivement l'évolution de la couche limite laminaire ou turbulente.

II. - ZONE A FAIBLE PROFONDEUR. -- La vilesse à la fronlière de la couche limite vérifie $u_{1} \frac{d u_{1}}{d x}=g \sin i$. En général, la couche limite devient turbulente dans cette zone. Le mouvement est défini en aval par l'équation (13), que nous avons montré ètre pratiquement vérifié par (14).

III. - Point critrovi. - Ce sera en général dans cette zone que la couche limite atteindra une hauteur égale à la profondeur de l'écoulement. Les equations foumissant la posilion du point critique sont étudiées au chapitre III, paragraphe 4. L'eau blanche est susceptible de se produire au point critique; nous tenterons dans la troisieme partie d'établir le critère qui détermine son apparition.

IV. - EN AVAI DU poINT critrous. - Lorsque l'eau blanche n'apparait pas, l'évolution du mouvement est étudiée au chapitre III, paragraphe 7. En général, la profondeur décroît et tend vers la profondeur du régime uniforme. Les applications présentées dans la seconde partie préciseront d'ailleurs ces différents points.

(A suivre.)

\section{BIBLIOGRAPHIE}

1. LANE (E. W.). - Entrainment of air in swiftly flowing water. Civ. Eng., vol. 9, $n^{\circ}$ 2, fév. 1939, p. 89.

2. HaLI (S.). - The influence of air entrainment on flow in steep chutes. Proc. of the III Hyd. Conf., Univ. of Iowa, 1946 , p. 298.

3. Gumensky (G.B.). - Air entrained in fast water affects design of training walls and stilling basins. Civ. Eng., vol. $19, \mathrm{n}^{\circ} 12$, déc. 1949 , p. 35.

4. Durand (W.F.). - Flow of water in channels under steep gradients. Trans, A.S.M.E., vol. $62, \mathrm{n}^{\circ} 1$, janvier 1940 , p. 9.

5. Schoklitsch (A.). - Stauraumberlandung und Kolkabwehr. Wien, Julius Springer, 1935, p. 161.

6. Escande (L.). - Compléments d'Hydranlique, $1^{\text {re }}$ partie. 'Toulouse, Ed. Privat, p. 78.

7. Codnstein (S.). - Modern developments in fluid dynamics. Oxford, Clarendon Press, 1938, p. 119.
8. Vox Mises. - Zeitschr. f. angew, Math. u. Mecha., vol. 7,1927, p. 425 .

9. Von KarmaN (T.). - Zeitschr. f. angew. Math. u. Mecha., vol. 1,1921 , p. 235

10. Pohllhuses, - Ibid., p. 256.

11. Pohlifauses. - Ibid., p. 257.

12. Rouse (H.). - Fluid mechanics for hydraulic Engineers. New-York and London, Mac Graw-Hill, Book Co., 1938, p. 197.

13. Prandit (L.). - Werft. Reederei. Hafen, vol. 14, 1934, p. 1.

14. Conolet (R.). - C. R. Acad. Sc., 21 juin 1948, t. 226, p. 2049 .

15. Bakmmetefr (B.). - Mécanique de l'écoulement turbulen't des fluides. Paris, Dunod, 1941, p. 77.

16. Goldosteln (S.). - op. cit., p. 326.

17. Rovse (H.) - Op. cit., p. 291.

18. Goldsteri (S.). - Op. cit., p. 329. 